\title{
O enfermeiro na prevenção do extravasamento de agentes antineoplásicos: uma revisão de literatura
}

O extravasamento de antineoplásicos consiste na infiltração do agente anticâncer no espaço extracelular e nos tecidos circundantes, devido à administração do medicamento fora do vaso sanguíneo, podendo causar reações severas no tecido, sendo primordial a prevenção desse evento, proporcionando para o paciente um tratamento seguro e eficaz, bem como a não incidência de danos. Este estudo teve como objetivo identificar nas produções cientificas as medidas preventivas diante do extravasamento de agentes antineoplásicos, e analisar os principais cuidados de enfermagem recomendados. Trata-se de uma revisão integrativa utilizando as bases de dados LILACS, PUBMED e BDENF para a busca dos estudos. Dentre as prevenções associadas ao extravasamento de agentes antineoplásicos, os cuidados de enfermagem mais predominantes foram a necessidade do treinamento da equipe de enfermagem, o estabelecimento de protocolos e diretrizes institucionais, a monitorização constante do local perfurado, a punção de veias grandes e calibras no antebraço e a utilização de cateter de calibre pequeno. Desta forma considera-se que os eventos de extravasamento podem estar associados à falta de treinamento e conhecimento científico técnico da equipe de enfermagem, um cuidado de enfermagem adequado podem prevenir este acontecimento e promover uma hospitalização segura, sendo indispensável à atuação da equipe de enfermagem, aplicando cuidados de qualidade para a prevenção deste cenário.

Palavras-chave: Antineoplásicos; Extravasamento de materiais terapêuticos e diagnósticos; Cuidados de enfermagem.

\section{The nurse in the prevention of antineoplastic agents extravasation: a literature review}

The extravasation of antineoplastic agents consists of the infiltration of the anticancer agent in the extracellular space and surrounding tissues, due to the administration of the drug outside the blood vessel, which can cause severe reactions in the tissue. It is essential to prevent this event, providing the patient with a safe and secure treatment effective, as well as the non-incidence of damage. This study aimed to identify, in scientific productions, preventive measures against the extravasation of antineoplastic agents, and to analyze the main recommended nursing care. This is an integrative review using LILACS, PUBMED and BDENF databases to search for studies. Among the preventions associated with the extravasation of antineoplastic agents, the most prevalent nursing care was the need to train the nursing staff, the establishment of institutional protocols and guidelines, constant monitoring of the perforated site, puncture of large veins and calipers in the forearm and the use of a small caliber catheter. Thus, it is considered that extravasation events may be associated with a lack of training and scientific and technical knowledge of the nursing team, adequate nursing care can prevent this event and promote safe hospitalization, being essential the performance of the nursing team, applying quality care to prevent this scenario.

Keywords: Antineoplastics; Extravasation of therapeutic and diagnostic materials; Nursing care.

Topic: Enfermagem Geral

Reviewed anonymously in the process of blind peer

Milena do Nascimento Vieira (ib)

Universidade de Sorocaba, Brasil

http://lattes.cnpq.br/8506527165765667

http://orcid.org/0000-0002-9596-6283

mileninhanvieira@gmail.com

Clayton Gonçalves de Almeida

Universidade de Sorocaba, Brasil

http://lattes.cnpq.br/6493791537446598

http://orcid.org/0000-0003-2959-3965

cgalmeida11@gmail.com

Seilla Siedler Tavares (iD

Universidade de Sorocaba, Brasil

http://orcid.org/0000-0002-3949-0102

sheilla.tavares@prof.uniso.br
Received: 17/06/2021

Approved: 19/07/2021
Leandro Aparecido Souza

Universidade de Sorocaba, Brasil

http://lattes.cnpq.br/6090315154831086

http://orcid.org/0000-0001-8828-9918

leandroapsouza14@gmail.com

Irineu Cesar Panzeri Contini

Universidade de Sorocaba, Brasil

http://lattes.cnpq.br/3751316399780774

http://orcid.org/0000-0002-7489-5527

irineu.contini@prof.uniso.br

\section{Referencing this:}

VIEIRA, M. N.; ALMEIDA, C. G.; TAVARES, S. S.; SOUZA, L. A.; CONTINI, I. C. P.. O enfermeiro na prevenção do extravasamento de agentes antineoplásicos: uma revisão de literatura. Scire Salutis, v.11, n.3, p.54-60, 2021. DOI: http://doi.org/10.6008/CBPC2236$\underline{9600.2021 .003 .0008}$ 


\section{INTRODUÇÃO}

A incidência da neoplasia maligna aumentou $20 \%$ no mundo na última década, tornando-se um problema de saúde pública mundial. A cirurgia, radioterapia e quimioterapia são as modalidades terapêuticas mais utilizadas para o tratamento do carcinoma, onde a seleção depende do tipo de câncer, do estadiamento e da idade do paciente (MELO et al., 2020; DIAS et al., 2019). O tratamento quimioterápico atua no ciclo e na divisão celular através das substâncias químicas, diante das drogas antineoplásicas, sendo administrada por acessos venosos periféricos ou centrais, necessitando de profissionais habilitados para a punção, pois os pacientes que realizam o tratamento por um período prolongado, possuem fragilidade vascular e cutânea, desgaste progressivo da rede venosa e trombocitopenia (DIAS et al., 2019; SCHNEIDER et al., 2011).

O tratamento intravenoso pode trazer consigo alguns eventos adversos, tendo o extravasamento como um deles, sendo de grande preocupação, pois pode levar ao dano tecidual devido a presença de antineoplásicos no tecido. O extravasamento consiste na infiltração do agente anticâncer no espaço extracelular e nos tecidos circundantes, devido a administração do medicamento fora do vaso sanguíneo. Ele pode causar reações severas no tecido, como a irritação e formação de vesículas, as lesões podem progredir levando a necrose tecidual, podendo atingir tendões, ligamentos, nervos e ossos, causando dor extrema e perda funcional do membro (OKUDA et al., 2017; GOZZO et al., 2017).

O enfermeiro está à frente deste processo, visto que é o profissional responsável por administrar a quimioterapia, de acordo com a Resolução n. 210, de 01 de julho de 1998 do Conselho Federal de Enfermagem (COFEN), entretanto a Oncology Nursing Society (ONS) relata que a administração de antineoplasicos deve ser feita exclusivamente pelos enfermeiros oncologistas. A prevenção desse evento é fundamental, proporcionando para o paciente um tratamento seguro e eficaz, bem como a não incidência de danos (SCHNEIDER et al., 2011; GOZZO et al., 2017).

Como um meio de prevenir a ocorrência do extravasamento dos agentes antineoplásicos, é indispensável o conhecimento da classificação das drogas a serem utilizadas, sendo subclassificadas em vesicantes ou irritantes, podendo ser agressivos para o vaso sanguíneo. Bem como, o conhecimento da equipe, para que sejam capacitados e qualificados para a aplicação das técnicas para a manipulação do cateter (GOZZO et al., 2017).

\section{METODOLOGIA}

Trata-se de uma revisão integrativa da literatura, seguindo as etapas descritivas, a fim de obter um melhor entendimento sobre o tema do trabalho baseado nos estudos anteriores. Sendo elas: 1) identificação do tema e seleção da hipótese ou questão de pesquisa; 2) estabelecimento de critérios para inclusão e exclusão de estudos/amostragem ou busca na literatura; 3) definição das informações a serem extraídas dos estudos selecionados/ categorização dos estudos; avaliação dos estudos incluídos; 4) interpretação dos resultados; 5) apresentação da revisão/síntese do conhecimento (ERCOLE, 2014).

Para a busca dos artigos, foram utilizadas as bases de dados: PUBMED, Literatura Latino-americana 
e do Caribe em Ciências da Saúde (LILACS) e Base de dados em Enfermagem (BDENF), utilizando os descritores em português nas bases de dados BDENF e LILACS: antineoplásicos; extravasamento de materiais terapêuticos e diagnósticos; cuidados de enfermagem. E os descritores em inglês na base de dados PUBMED: antineoplasics; extravasation of therapeutic and diagnostic materials nursing care, utilizando AND como booleano de escolha.

A pergunta de pesquisa norteadora para esta revisão integrativa foi: Quais os meios de prevenção da ocorrência do extravasamento de agentes antineoplásicos e os cuidados de enfermagem recomendados? Na seleção das produções científicas foram utilizados alguns critérios de inclusão, primeiramente considerou-se o idioma dos artigos inglês, espanhol e português, tendo como delimitação de data publicada entre 2010 a 2020. Posteriormente foram selecionados a partir do título e resumo respondendo o tema de interesse, e por último os artigos foram avaliados pela análise do conteúdo. As publicações não equivalentes aos critérios de inclusão foram excluídas. Na Figura 1 mostra o processo da busca representado em fluxograma adaptado do Preferred Reporting Items for Systemati Reviews and meta- analyses (PRISMA). Foram identificados 252 artigos por meio da busca nas bases de dados, foram excluídas 20 publicações duplicadas, totalizando 232 para a leitura do título e resumo. Ao final, foram excluídos 70 artigos, pois exploravam assuntos fora do objetivo proposto no trabalho, sendo incluídos 9 artigos para a realização deste trabalho.

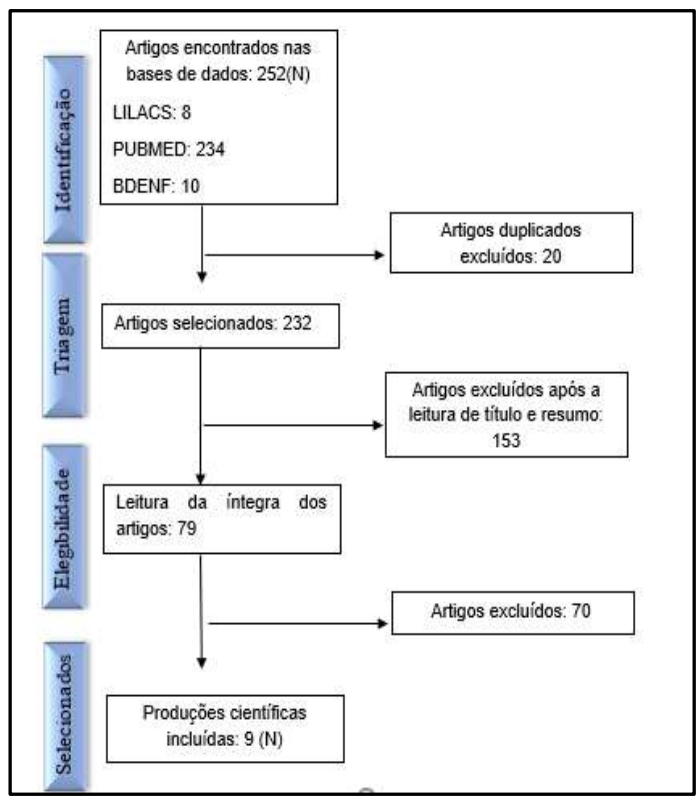

Figura 1: Fluxograma PRISMA da seleção de artigos nas bases de dados.

\section{RESULTADOS E DISCUSSÃO}

A seguir no Quadro 1 estão as produções científicas desta revisão integrativa em ordem decrescente de publicação com ano de publicação/autores, idioma da pesquisa, título do artigo e objetivo dos respectivos trabalhos. Os estudos foram identificados como estudo 1 (E1) e assim respectivamente.

Diante das prevenções encontradas na literatura, 7 (77,7 \%) dos artigos relataram a prevalência da necessidade do treinamento da equipe de enfermagem para realizar o procedimento, a educação continuada, sendo indispensável para os profissionais, visto que é fundamental o conhecimento teórico- 
prático para a segurança do paciente e que a prevenção de extravasamento seja sempre a primeira opção para o atendimento do paciente. Na Tabela 1 estão os principais cuidados de enfermagem encontrados na literatura, com representação porcentual.

Quadro 1: Produções científicas com informações sobre ano de publicação, autores, idioma e objetivo dos estudos. Sorocaba, Brasil, 2020

\begin{tabular}{|c|c|c|c|c|}
\hline $\begin{array}{l}\text { Estudo } \\
\text { (E) }\end{array}$ & Título do artigo & Autores/ano & Idioma & Objetivo \\
\hline E1 & $\begin{array}{l}\text { Construção e avaliação de bundle } \\
\text { frente ao extravasamento de } \\
\text { antineoplásicos: estudo metodológico }\end{array}$ & $\begin{array}{l}\text { Melo et al., } \\
2020\end{array}$ & Português & $\begin{array}{l}\text { Construir e avaliar o conteúdo de um bundle de prevenção } \\
\text { e condutas frente ao extravasamento de agentes } \\
\text { antineoplásicos em pacientes oncológicos adultos. }\end{array}$ \\
\hline E2 & $\begin{array}{l}\text { Prevenção e conduta contra o } \\
\text { extravasamento da quimioterapia } \\
\text { antineoplásica: uma revisão do escopo }\end{array}$ & $\begin{array}{l}\text { Melo et al., } \\
2020\end{array}$ & Português & $\begin{array}{l}\text { Identificar e sintetizar evidências científicas sobre } \\
\text { prevenção e manejo do extravasamento de agentes } \\
\text { antineoplásicos em pacientes adultos por enfermeiros. }\end{array}$ \\
\hline E3 & $\begin{array}{l}\text { Padrões de cuidados em prevenção e } \\
\text { tratamento de extravasamento de } \\
\text { antineoplásicos baseado em evidências } \\
\text { clínicas }\end{array}$ & $\begin{array}{l}\text { Dias et al., } \\
2019\end{array}$ & Português & $\begin{array}{l}\text { Descrever padrões de cuidados em prevenção e } \\
\text { tratamento de extravasamento de antineoplásicos baseado } \\
\text { em evidências clínicas. }\end{array}$ \\
\hline E4 & $\begin{array}{l}\text { Conhecimento dos Profissionais de } \\
\text { Enfermagem sobre Segurança do } \\
\text { Paciente Oncológico em Quimioterapia }\end{array}$ & $\begin{array}{l}\text { Costa et al., } \\
2019\end{array}$ & Português & $\begin{array}{l}\text { Avaliar o conhecimento dos profissionais de enfermagem } \\
\text { quanto à segurança do paciente oncológico em tratamento } \\
\text { quimioterápico }\end{array}$ \\
\hline E5 & $\begin{array}{l}\text { Skin Necrosis Due to the Extravasation } \\
\text { of Irritant Anticancer Agents }\end{array}$ & $\begin{array}{l}\text { Okuda et al., } \\
2018\end{array}$ & Inglês & $\begin{array}{l}\text { Relatar experiência com um caso de necrose da pele devido } \\
\text { ao extravasamento de dois agentes anticâncer irritantes, } \\
\text { gemcitabina e cisplatina. }\end{array}$ \\
\hline E6 & $\begin{array}{l}\text { Evaluation of the Predictive Validity of } \\
\text { Thermography in Identifying } \\
\text { Extravasation With Intravenous } \\
\text { Chemotherapy Infusions }\end{array}$ & $\begin{array}{l}\text { Matsui et al., } \\
2017\end{array}$ & Inglês & $\begin{array}{l}\text { Examinar a validade da termografia na previsão do } \\
\text { extravasamento após a administração de um agente } \\
\text { antineoplásico por infusão intravenosa e demonstrar as } \\
\text { limitações da termografia no diagnóstico do } \\
\text { extravasamento. }\end{array}$ \\
\hline E7 & $\begin{array}{l}\text { Conhecimento da equipe de } \\
\text { enfermagem acerca da prevenção e } \\
\text { manejo de extravasamento de drogas } \\
\text { quimioterápicas }\end{array}$ & $\begin{array}{l}\text { Gozzo et al., } \\
2017\end{array}$ & Português & $\begin{array}{l}\text { Identificar o conhecimento dos profissionais da equipe de } \\
\text { Enfermagem acerca da prevenção e manejo do } \\
\text { extravasamento de drogas quimioterápicas durante o } \\
\text { tratamento oncológico. }\end{array}$ \\
\hline E8 & $\begin{array}{l}\text { Extravasation of Antineoplastic Agents: } \\
\text { Prevention and Treatments }\end{array}$ & $\begin{array}{l}\text { Boschi et al., } \\
2012\end{array}$ & Inglês & $\begin{array}{l}\text { Este artigo fornece uma revisão da literatura atual sobre } \\
\text { recomendações sobre prevenção e tratamento do } \\
\text { extravasamento de agentes antineoplásicos. }\end{array}$ \\
\hline E9 & $\begin{array}{l}\text { Extravasamento de drogas } \\
\text { antineoplasicas: avaliação do } \\
\text { conhecimento da equipe de } \\
\text { enfermagem }\end{array}$ & $\begin{array}{l}\text { Schneider et } \\
\text { al., } \\
2011\end{array}$ & Português & $\begin{array}{l}\text { Avaliar o conhecimento da equipe de enfermagem de um } \\
\text { ambulatório de quimioterapia adulto sobre o } \\
\text { extravasamento de drogas antineoplasicas }\end{array}$ \\
\hline
\end{tabular}

Tabela 1: Principais prevenções para extravasamento de antineoplásico identificados na literatura.

\begin{tabular}{|c|c|c|}
\hline Cuidados de Enfermagem & $\mathbf{N}$ & $\%$ \\
\hline Treinamento da equipe de enfermagem & 7 & 77,7 \\
\hline Implementação de protocolos e diretrizes & 5 & 55,5 \\
\hline Monitoração constante do local perfurado e o paciente & 4 & 44,4 \\
\hline Puncionar veias grandes e calibrosas no antebraço & 4 & 44,4 \\
\hline O calibre do dispositivo deve ser respectivo ao vaso & 4 & 44,4 \\
\hline Utilizar cateter de pequeno calibre & 4 & 44,4 \\
\hline Evitar punção em membros com perda de sensibilidade & 3 & 33,3 \\
\hline Evitar punção nos membros inferiores & 3 & 33,3 \\
\hline Lavar a veia com 10 a $20 \mathrm{ml}$ soro fisiológico após a infusão de quimioterápico ou entre suas infusões & 3 & 33,3 \\
\hline Evitar dispositivo rígido & 3 & 33,3 \\
\hline Evitar o uso de veias puncionadas a mais de 24 horas & 3 & 33,3 \\
\hline Orientar a verbalização de sinais e sintomas & 2 & 22,2 \\
\hline Conferir retorno venoso e após a punção, lavar com $10 \mathrm{ml}$ de salina normal & 2 & 22,2 \\
\hline Orientar paciente quanto ao risco & 2 & 22,2 \\
\hline A fixação não deve ser em excesso para não prejudicar a visibilidade & 2 & 22,2 \\
\hline Confirmação dos dados do paciente & 1 & 11,1 \\
\hline
\end{tabular}

A necessidade da existência de protocolos e diretrizes foram encontradas em $5(55,5)$ artigos, a padronização dos procedimentos uniformiza o tipo de tratamento para oferecer ao paciente, diminui o risco de erros e de eventos adversos. O enfermeiro deve monitorar o paciente e o local puncionado, a fim 
de verificar possíveis sinais de extravasamento, assim como encontrado em $4(44,4 \%)$ artigos. Relacionado ao paciente, ele deve ser orientado quanto ao risco de extravasamento $(22,2 \%)$ e orientar a verbalização na presença de sinais e sintomas $(22,2 \%)$ relacionados ao extravasamento, para que na presença deles o tratamento terapêutico seja interrompido imediatamente e não ocorra danos maiores.

É indispensável os cuidados na escolha do dispositivo a ser utilizado, o calibre deve corresponder ao vaso sanguíneo do paciente $(44,4 \%)$, evitar dispositivos rígidos $(33,3 \%)$, e utilizar cateteres de pequeno calibre no paciente assim como relatado em $4(44,4 \%)$ artigos. A avaliação e cuidados com o local da punção devem estar presente na rotina dos profissionais, é recomendado puncionar veias grandes e calibrosas no antebraço do paciente $(44,4 \%)$, evitar membros com perda de sensibilidade e membros inferiores $(33,3 \%)$, e evitar o uso de veias puncionadas a mais de 24 horas. $2(22,2 \%)$ artigos relatam a necessidade de verificar o retorno venoso e após a punção lavar com $10 \mathrm{ml}$ de salina normal, 3 (33,3\%) artigos indica a lavagem da veia com 10 a $20 \mathrm{ml}$ de soro fisiológico após a infusão de quimioterápicos ou entre suas infusões. A fixação do cateter deve ser exposta assim como encontrados em $2(22,2 \%)$ dos artigos, não prejudicando a visibilidade da fixação, bem como a necessidade de confirmar os dados do paciente em $1(11,1 \%)$ dos estudos.

Quadro 2: Prevenção para o extravasamento de antineoplásicos.

\begin{tabular}{|l|l|}
\hline Prevenção para o extravasamento de antineoplasicos \\
\hline Dispositivo & $\begin{array}{l}\text { Orientar o paciente quanto ao risco de extravasamento } \\
\text { Orientar a verbalização de sinais e sintomas relacionados ao extravasamento }\end{array}$ \\
\hline Local de punção & $\begin{array}{l}\text { O calibre do dispositivo corresponder ao vaso sanguíneo do paciente } \\
\text { Evitar dispositivo rígido } \\
\text { Utilizar cateteres de pequeno calibre }\end{array}$ \\
& $\begin{array}{l}\text { Puncionar veias grandes e calibrosas no antebraço } \\
\text { Evitar puncionar membros com perda de sensibilidade } \\
\text { Conferir retorno venoso e após a punção lavar com } 10 \text { ml de salina normal } \\
\text { Evitar puncionar nos membros inferiores } \\
\text { Lavar a veia com soro fisiológico após a infusão de quimioterápico ou entre suas infusões } \\
\text { Evitar o uso de veias puncionadas a mais de } 24 \text { horas } \\
\text { A fixação não deve ser excessiva, para não prejudicar a visibilidade }\end{array}$ \\
\hline Equipe de enfermagem & $\begin{array}{l}\text { Treinamento permanente dos profissionais, para uma capacitação de técnicas } \\
\text { Padronizar técnicas de punção, através de protocolos e diretrizes } \\
\text { Confirmar os dados do paciente, prevenindo possíveis erros } \\
\text { Monitorar constantemente o local perfurado e o paciente. }\end{array}$ \\
\hline
\end{tabular}

No Quadro 2 apresenta-se os meios de prevenção relacionado a cada categoria. A prevenção do extravasamento é a melhor medida de assistência a ser ofertada, o treinamento e educação continuada é indispensável para uma boa prática, assegurando uma segurança do paciente adequada (MELO et al., 2020). O profissional deve possuir conhecimento sobre a classificação, toxicidade dermatológica local e o mecanismo de ação das drogas, os métodos eficazes de prevenção, os sinais e sintomas, os cuidados necessários e as possíveis complicações (SCHNEIDER et al., 2011). A implementação de protocolos institucionais possibilita a sistematização e unificação das ações, baseada em evidências, é de grande importância padronizar as técnicas nas instituições (DIAS et al., 2019).

É inúmeros os fatores contribuintes para o extravasamento, o condicionamento do paciente pode influenciar neste fator, pois ele pode ser mais propenso a medida que apresenta disfunção endotelial como idade avançada, diabete mellitus, hipertensão, dislipidemia, aterosclerose, insuficiência renal crônica, fluxo 
sanguíneo comprometido, ou em casos de pacientes pediátricos que são incapazes de relatar e descrever a dor, e pela inexperiência da equipe de percepção dos sinais e sintomas (DIAS et al., 2019).

Outros fatores de risco relacionados são alterações das veias com fragilidade e esclerose, local inadequado da punção, escolha de dispositivo para a punção inadequada, alterações no membro com condições prejudiciais. Os cateteres venosos centrais oferecem risco para o extravasamento, pois sua extensão e ponta podem se curvar ou se romper, tendo a formação de trombo em sua ponta (DIAS et al. 2019; SCHNEIDER et al., 2011). O papel da enfermagem no extravasamento é de extrema importância, pois ele é responsável pela punção venosa, administração dos medicamentos e pelo tratamento das intercorrências. O cuidado de enfermagem inclui planejar e realizar as intervenções necessárias (MELO et al., 2020; SCHNEIDER et al., 2011).

A escolha do cateter deve ser multifatorial, a escolha da veia é preferencialmente calibrosa e não tortuosas, fino calibre, esclerosada, moveis, e que sofreram múltiplas punçoes anteriores, o dispositivo deve ser compatível a veia selecionada. Um método preventivo é a lavagem do acesso com 10 a $20 \mathrm{ml}$ de solução fisiológica de $0,9 \%$ entre as infusões, o enfermeiro deve estar atento ao aparecimento de sintomas como edema, rubor, dor e infusão lenta (DIAS et al., 2019).

O extravasamento pode causar necrose ao tecido lesado, causando limitação nas atividades diárias do paciente, e tardiamente pode levar a dificuldade na condução da quimioterapia, podendo até mesmo prejudicar o prognostico. É necessária cautela no tempo de infusão da droga e a linha intravenosa, um tempo de 60 minutos ou mais está associado a uma incidência de mielossupressão e comprometimento da função hepática, é indicado que o medicamento seja administrado em 30 minutos (OKUDA et al., 2018).

\section{CONCLUSÕES}

A quimioterapia é um dos tratamentos terapêuticos para a neoplasia, entretanto, a sua administração deve ser adequada de modo que não ocorra eventos adversos, como o extravasamento. 0 extravasamento pode causas lesões importantes e irreversíveis nos tecidos, deste modo a prevenção é a estratégia mais eficaz a ser adotada pela equipe de enfermagem. A equipe de enfermagem deve ser capacitada para manipular os agentes antineoplásicos, se faz necessário o estabelecimento de protocolos institucional e um programa de educação continuada dos profissionais, de modo a suprir o conhecimento sobre a prevenção, identificação e manejo do extravasamento de antineoplásico.

Os eventos de extravasamento podem estar associados a falta de treinamento e conhecimento científico e técnico da equipe de enfermagem, um cuidado de enfermagem adequado podem prevenir este acontecimento e promover uma hospitalização e tratamento terapêutico seguro.

\section{REFERÊNCIAS}

BOSCHI, R.; ROSTAGNO, E.. Extravasation of antineoplastic agents: prevention and treatments. Pediatric reorts, v.4, n.3, 2012. DOI: https://doi.org/10.4081/pr.2012.e28

COSTA, A. G.; COSTA, M. S. C. R.; FERREIRA, E. S.; SOUZA, P. C.; SANTOS, M. M.; LIMA, D. E. O. B.; RAMOS, A. M. P. C. Conhecimento dos profissionais de enfermagem sobre segurança do paciente oncológico em quimioterapia. Revista Brasileira de cancerologia, v.65, n.1, 2019. DOI: https://doi.org/10.32635/2176-9745.RBC.2019v65n1.274

DIAS, S. R. S.; AVELINO, F. V. S. D.; MOURA, E. C. C.; COSTA, J. P.. Padrões de cuidados em prevenção e tratamento de extravasamento de antineoplásicos baseado em evidências 
clínicas. Revista enfermagem atual in derme, v.87, n.25, 2019. DOI: https://doi.org/10.31011/reaid-2019-v.87n.especial-art.173

ERCOLE, F. F.; MELO, L. S.; ALCOFORADO, C. L. G. C.. Revisão integrativa versus revisão sistemática. Revista Mineira de Enfermagem, v.18, n.1. DOI: https://doi.org/10.5935/1415$\underline{2762.20140001}$

GOZZO, T. O.; SANTOS, L. A. C.; CRUZ, L. A. P.. Conhecimento da equipe de enfermagem acerca da prevenção e manejo de extravasamento de drogas quimioterápicas. Revista de enfermagem, v.11, n.12, p.4789-4797, 2017. DOI: https://doi.org/10.5205/1981-8963-v11i12a15191p4789$\underline{4797-2017}$

MATSUI, Y.; MURAYAMA, R.; TANABE, H.; OE, M.; MOTOO, Y.; WAGATSUMA, T.; MICHIBUCHI, M.; KINOSHITA, S.; SAKAI, K.; KONYA, C.; SUGAMA, J.; SANADA, H.. Evaluation of the predictive validity of thermography in identifying extravasation with intravenous chemotherapy infusions. Journal of infusion nursing, v.40, n.6, p.367-374, 2017. DOI: https://dx.doi.org/10.1097\%2FNAN.0000000000000250
MELO, J. M. A.; OLIVEIRA, P. P.; RODRIGUES, A. B.; SOUZA, R. S.; FONSECA, D. F.; GONTIJO, T. F.; SILVEIRA, E. A. A.. Construção e avaliação de bundle frente ao extravasamento de antineoplásicos: estudo metodológico. Acta paul. Enferm, v.33, 2020. DOI: https://doi.org/10.37689/actaape/2020ao0075

MELO, J. M. A.; OLIVEIRA, P. P.; SOUZA, R. S.; FONSECA, D. F.; GONTIJO, T. F.; RODRIGUES, A. B.. Prevention and conduct against the extravasation of antineoplastiic chemotherapy: a scoping review Ver. Bras. Enferm, v.73, n.4, 2020. DOI: DOI: https://doi.org/10.1590/0034-7167-2019-0008

OKUSA, H.; MASATSUGU, A.; SIJIMAYA, T.; ARAI, R.. Skin necrosis due to the extravasation of irritant anticancer agents. Internal medicine, v.57, n.5, 2018. DOI: https://dx.doi.org/10.2169\%2Finternalmedicine.9329-17

SCHNEIDER, F.; PEDROLO, E.. Extravasamento de drogas antineoplásicas: avaliação do conhecimento da equipe de enfermagem. Revista Mineira de Enfermagem, v.15, n.4, p. 522-529, 2011 DOI:

https://doi.org/10.25248/reas.e1177.2019

A CBPC - Companhia Brasileira de Produção Científica (CNPJ: 11.221.422/0001-03) detém os direitos materiais desta publicação. Os direitos referem-se à publicação do trabalho em qualquer parte do mundo, incluindo os direitos às renovações, expansões e disseminações da contribuição, bem como outros direitos subsidiários. Todos os trabalhos publicados eletronicamente poderão posteriormente ser publicados em coletâneas impressas sob coordenação da Sustenere Publishing, da Companhia Brasileira de Produção Científica e seus parceiros autorizados. Os (as) autores (as) preservam os direitos autorais, mas não têm permissão para a publicação da contribuição em outro meio, impresso ou digital, em português ou em tradução. 